\title{
Growth Versus Environment in Dynamic Models of Capital Accumulation
}

\author{
TOICHIRO ASADA* \\ Faculty of Economics, Chuo University, 742-1 Higashinakano, Hachioji, Tokyo 192-0393, Japan, \\ and Department of Economics, University of Bielefeld, Germany \\ (Revised 14 April 2001)
}

\begin{abstract}
In this paper, we study the economic implications of the trade off between growth and environment in the context of dynamic models of capital accumulation. The collective solution is formulated in terms of dynamic optimization of the central planner, and the decentralized solution is formulated in terms of differential game between workers and capitalists. We compare the economic properties of two solutions.
\end{abstract}

Keywords: Growth; Environment; Capital accumulation; Dynamic optimization; Differential game; Open loop Nash solution; Feedback Nash solution

\section{INTRODUCTION}

Since the 1970s, a lot of works on environmental economics from the dynamic perspectives have been accumulated. Early pioneering examples are Keeler et al. (1971), Mäler (1974), and Clark (1976). More recent contributions are, among others, Forster (1980), Uzawa (1995), Hartl and Kort (1996) and Hettich (2000). They investigated the economic implications of the government's environmental policies by applying mathematical techniques of dynamic optimization. Usually, models in such a tradition are formulated in terms of single agent optimization problems. Recently, however, analyses of the conflict of interests between several agents in terms of (dynamic and static) games in the context of environmental economics have been developed. We can refer to Dockner et al. (1989), Dockner and van Long (1993), Mäler and de Zeeuw (1998), Stimming (1999) and Uzawa (1999) as important examples. They developed the gametheoretical approaches, which can analyze the conflict of interests between fishermen, between government and private sector, or between countries, and the effects of the cooperative and noncooperative behaviors of economic agents on the global environment were investigated. Dynamic games (in particular, differential games) are powerful tools to study such a theme. $\dagger$

In this paper, we also consider a game-theoretical approach of the environmental economics from the dynamic perspective, but we put much emphasis on a rather neglected aspect in the environmental economics. We focus on the conflict of interests between two major classes in the capitalist economy, i.e. workers and capitalists. We introduce the environmental factor into the analytical framework of the "differential game of capitalism" which was initiated by Lancaster (1973)'s pioneering paper and developed by Hoel (1978), Pohjola (1983), Mehrling (1986) and Ishigaki (1994) and investigate the economic implications of the solutions.

This paper is organized as follows. In second section, we introduce the environmental factor into a very simple model of capital accumulation in which the rate of economic growth is determined endogenously, and the "collective solution", which means the solution of the central planner's dynamic optimization problem, is studied by means of the optimal control technique. In third section, we formulate a "differential game of capitalism" between workers and capitalists, and consider the open loop Nash solution as a typical solution of such a

\footnotetext{
*Corresponding author. E-mail: asada @tamacc.chuo-u.ac.jp

$\dagger$ As for the survey of the differential game approach to the transboundary pollution problem, see Inoue (1998).

${ }^{\ddagger}$ As for the differential game of capitalism, see also Asada (1997).
} 
game." It will be shown that in general such a solution is different from the collective solution, and we obtain some paradoxical results. For example, it is possible that the shift of the workers' preference from the economic growth to the environment may in fact raise the rate of economic growth.

\section{THE COLLECTIVE SOLUTION}

Let us consider the (hypothetical) planned economy in which the social welfare functional of the central planner is expressed as

$$
W=\int_{0}^{\infty}\{U(C / N) N-V(P)\} \exp (-\rho t) \mathrm{d} t
$$

where $C$ is the total real consumption, $N$ is the labor employment, $P$ is the stock of pollution, $\rho$ is the rate of discount which is assumed to be a positive constant, and it is assumed that

$$
\begin{aligned}
U^{\prime}(C / N) & >0, \quad U^{\prime \prime}(C / N)<0, \quad V^{\prime}(P)>0, \\
V^{\prime \prime}(P) & \geqq 0 .
\end{aligned}
$$

As for the technology, we assume the fixed technological coefficients. That is to say,

$$
Y / K=a>0, \quad N / K=b>0,
$$

where $a$ is the output-capital ratio and $b$ is the labor-capital ratio, and they are assumed to be constant. ${ }^{\S}$

We also assume that

$$
\begin{gathered}
\dot{K}=I=S=s Y \quad(0 \leqq \mathrm{~s} \leqq 1), \\
C=(1-s) Y
\end{gathered}
$$

where $I$ is the real investment, $S$ is the real saving, and $s$ is the saving rate which is to be controlled by the central planner.

Substituting Eq. (3) into Eqs. (4) and (5), we have

$$
\begin{gathered}
g \equiv \dot{K} / K=s a \\
C / N=(1-s)(a / b)
\end{gathered}
$$

where $g \equiv \dot{K} / K$ is the rate of capital accumulation (the rate of economic growth).

We further assume that the stock of pollution $(P)$ is proportional to the capital stock $(K)$, but $P$ also depends positively on the rate of economic growth $(g)$. Namely,

$$
P=\varphi(g) K ; \quad \varphi(0)>0, \quad \varphi^{\prime}(g)>0, \quad \varphi^{\prime \prime}(g) \geqq 0 .
$$

To simplify the analysis, we adopt the following specification of the functional forms.

$$
\begin{gathered}
U(C / N)=\log (C / N) \\
V(P)=\alpha P ; \quad \alpha>0 \\
\varphi(g)=\beta(1+g) ; \quad \beta>0
\end{gathered}
$$

In this case, we have

$$
\begin{gathered}
U(C / N) N-V(P)=\{\log (C / N)\} N-\alpha P \\
=[\log \{(1-s)(a / b)\}] b K-\alpha \beta(1+s a) K \\
=\{\log (1-s)+\log a-\log b-(\alpha \beta / b)(1+s a)\} b K \\
U(C / N) N-V(P) \equiv f(s ; \alpha \beta) K
\end{gathered}
$$

where

$$
\begin{aligned}
f(s ; \alpha \beta) \equiv & \{\log (1-s)+\log a-\log b-(\alpha \beta / b)(1 \\
& +s a)\} b .
\end{aligned}
$$

Now, we can formulate the optimal program of the central planner as follows.

$$
\operatorname{Max}_{s(t) \in[0,1]} \int_{0}^{\infty} f(s(t) ; \alpha \beta) K(t) \exp (-\rho t) \mathrm{d} t
$$

subject to

$$
\dot{K}(t)=s(t) a K(t), \quad K(0) \equiv K_{0}=\text { given. }>0 .
$$

To solve this problem mathematically, we can make use of the Pontryagin's maximum principle."

The current value Hamiltonian $(H(t))$ in this system becomes

$$
H(t) \equiv f(s(t) ; \alpha \beta) K(t)+\lambda(t) s(t) a K(t)
$$

where $\lambda(t)$ is the costate variable. Then, we can write a set of necessary conditions for optimality as follows.

i)

$$
\operatorname{Max}_{s(t) \in[0,1]} H(t) \text { for all } t \geqq 0 .
$$

ii)

$$
\begin{aligned}
\dot{\lambda}(t) & =-\partial H(t) / \partial K(t)+\rho \lambda(t) \\
& =-f(s(t) ; \alpha \beta)+\{\rho-s(t) a\} \lambda(t) \text { for all } t \geqq 0 .
\end{aligned}
$$

iii)

$$
\lim _{t \rightarrow \infty} \lambda(t) \exp (-\rho t)=0 .
$$

\footnotetext{
"It will be shown in third section that in our model the open loop Nash solution is also the feedback Nash solution.

${ }^{8} Y$ is the real national income (real output) and $K$ is the real physical capital stock. It follows from Eq. (3) that $N=b K$, which implies that the labor employment is constrained by the existing capital stock, so that in general the full employment of labor will not be satisfied. We assume the labor-surplus economy in which the labor supply does not become a constraint of the production. Obviously, unemployment in our model is not the "Keynesian" unemployment which is due to insufficient effective demand but the "classical" unemployment which is due to insufficient capital stock.

"As for the Pontryagin's maximum principle, see, for example, Chiang (1992).
} 
It follows from Eq. (15) that

$$
\begin{aligned}
& \mathrm{d} H(t) / \mathrm{d} s(t)=\left\{f^{\prime}(s(t))+\lambda(t) a\right\} K(t) \\
= & {[-b /\{1-s(t)\}-\alpha \beta a+\lambda(t) a] K(t), } \\
= & -b /\{1-s(t)\}^{2}<0 \text { if } s(t) \in[0,1) .
\end{aligned}
$$

If we assume the internal solution, Eq. (16)(i) is reduced to $\mathrm{d} H(t) / \mathrm{d} s(t)=0$, i.e.

$$
\lambda(t)=(b / a) /\{1-s(t)\}+\alpha \beta \equiv \lambda(s(t))>0 .
$$

Substituting Eq. (19) into Eq. (16)(ii), we obtain

$$
\begin{aligned}
{[(b / a) /\{1-s(t)\}] \dot{s}(t)=} & -f(s(t) ; \alpha \beta)+\{\rho \\
& -s(t) a\} \lambda(s(t)) \\
& \equiv F(s(t))
\end{aligned}
$$

which is a differential equation with only variable, $s(t)$. We obtain

$$
\begin{aligned}
F^{\prime}(s(t)) & =-f^{\prime}(s(t))+\{\rho-s(t)\} \lambda^{\prime}(s(t))-a \lambda(s(t)) \\
= & \{\rho-s(t) a\}(b / a) /\{1-s(t)\}^{2}, \\
F(0)= & -f(0 ; \alpha \beta)+\rho \lambda(0) \\
= & \{-\log a+\log b+\rho / a\} b+\alpha \beta(1+\rho) \\
= & \{\log (b / a)+\rho / a\} b+\alpha \beta(1+\rho), \\
\lim _{s(t) \rightarrow 1} F(s(t))= & \left\{-\lim _{s(t) \rightarrow 1} \log \{1-s(t)\}-\log a\right. \\
& +\log b\} b+\alpha \beta(1+a)+(\rho-a) \\
& \times\left[\lim _{s(t) \rightarrow 1}(a / b) /\{1-s(t)\}+\alpha \beta\right] .
\end{aligned}
$$

Now, let us assume as follows.

\section{ASSUMPTION 1}

$$
\text { (i) } \rho>a . \quad \text { (ii) } F(0)<0 \text {. }
$$

Under Assumption 1(i) we obtain

$$
F^{\prime}(s(t))>0, \quad \lim _{s(t) \rightarrow 1} F(s(t))=+\infty .
$$

Under Assumption 1, we have the unique stationary solution $s^{*} \in(0,1)$ of Eq. (20) (see Fig. 1). It is clear that the stationary solution $s^{*}$ satisfies the "transversality condition" (Eq. (16) (iii)).

Now, we can summarize the main results of this section as the following two propositions.

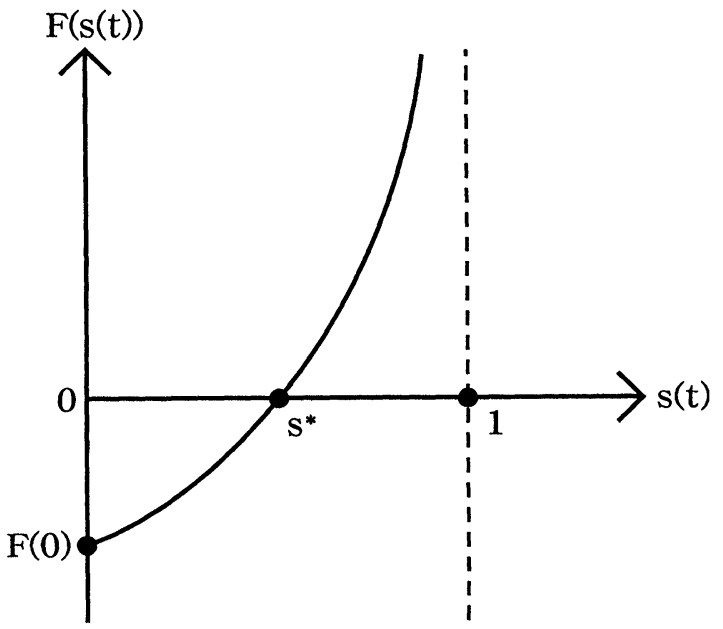

FIGURE 1 The stationary solution of Eq. (20).

Proposition 1 Under Assumption 1, the only optimal solution of the problem Eq. (14) is given by

$$
s(t)=s^{*} \text { for all } t \geqq 0,
$$

where $s^{*} \in(0,1)$ is the unique stationary solution of Eq. (20).

Proof See Appendix A.

\section{Proposition 2}

$$
\partial s^{*} / \partial(\alpha \beta)<0 .
$$

Proof We have

$$
\begin{aligned}
\partial F(s(t) ; \alpha \beta) / \partial(\alpha \beta) & =1+s(t) a+\{\rho-s(t) a\} \\
& =1+\rho>0 .
\end{aligned}
$$

Therefore, we have the following expression in view of Eq. (21) by totally differentiating the equation $F\left(s^{*} ; \alpha \beta\right)=0$.

$$
\begin{aligned}
& {\left[\left\{\rho-s^{*} a\right\}(b / a) /\left\{1-s^{*}\right)^{2}\right] \mathrm{d} s^{*}+(1+\rho) \mathrm{d}(\alpha \beta)} \\
& \quad=0 .
\end{aligned}
$$

Rearranging Eq. (28), we obtain

$$
\begin{aligned}
\mathrm{d} s^{*} / \mathrm{d}(\alpha \beta) & =-(a / b)(1+\rho)\left(1-s^{*}\right)^{2} /\left\{\rho-s^{*} a\right\} \\
& <0
\end{aligned}
$$

because $\rho-s^{*} a>\rho-a>0$ from Assumption $1 .^{\#}$

Proposition 1 says that the optimal rate of capital accumulation $\left(g^{*}\right)$ is endogenously determined, and it is

\footnotetext{
"More intuitive proof is as follows. The increase of $\alpha \beta$ induces the upward shift of the function $F(s(t))$ in Fig. 1 so that it induces the decrease of $s^{*}$.
} 
given by

$$
g^{*}=\dot{K} / K=s^{*} a>0 .
$$

$* *$

Proposition 2 implies that

$$
\partial g^{*} / \partial(\alpha \beta)<0
$$

in other words, the increase of the marginal disutility of pollution for the planner $(\alpha)$ or the increase of the pollution effect of economic growth $(\beta)$ will induce the decrease of the optimal rate of economic growth. Obviously, this is the reasonable conclusion.

\section{A DECENTRALIZED SOLUTION AS A DIFFERENTIAL GAME OF CAPITALISM}

Next, we shall consider the decentralized solution which reflects the institutional character of the capitalist economy. This approach tries to formalize the conflict of interests between workers and capitalists and the struggle over income distribution, capital accumulation, and the environmental pollution. We adopt the analytical framework of the "differential game of capitalism" which was introduced by Lancaster (1973) and others. ${ }^{\dagger \dagger}$

We assume that the workers' objective functional $\left(W_{1}\right)$ and the capitalists' objective functional $\left(W_{2}\right)$ are expressed as follows, respectively.

$$
\begin{gathered}
W_{1}=\int_{0}^{\infty}\left\{U_{1}\left(C_{W} / N\right) N-V_{1}(P)\right\} \exp (-\rho t) \mathrm{d} t \\
W_{2}=\int_{0}^{\infty} U_{2}\left(C_{K} / K\right) K \exp (-\rho t) \mathrm{d} t
\end{gathered}
$$

where $C_{W}=$ workers' real consumption, $C_{K}$ $=$ capitalists' real consumption, $N=$ labor employment, $K=$ real capital stock, $P=$ stock of pollution, and $\rho$ $=$ rate of discount which is common to both classes (positive constant).

We assume that

$$
\begin{aligned}
U_{1}^{\prime}\left(C_{W} / N\right)>0, \quad U_{1}^{\prime \prime}\left(C_{W} / N\right)<0, \\
V_{1}^{\prime}(P)>0, \quad V_{1}^{\prime \prime}(P) \geqq 0, \\
U_{2}^{\prime}\left(C_{K} / K\right)>0, \quad U_{2}^{\prime \prime}\left(C_{K} / K\right)<0 .
\end{aligned}
$$

In this formulation, we introduce the asymmetrical effect of the environmental pollution on the welfare of two classes. To stress the asymmetrical effect, we simply assume that the pollution affects only workers' welfare adversely, and it is assumed that the capitalists' welfare only depends on their own real consumption and real capital stock. ๆ

Eqs. (3) and (8) in the previous section are retained also in this section. However, in this section, we must modify Eqs. (4), (5) and (7) as follows:

$$
\begin{gathered}
\dot{K}=I=S=s_{k} \pi=s_{k}(1-z) Y=s_{k}(1-z) a K \\
\left(0 \leqq \mathrm{z} \leqq 1, \quad 0 \leqq s_{k} \leqq 1\right) \\
C_{W}=z Y=z a K \\
C_{W} / N=z(a / b) \\
C_{K}=\left(1-s_{k}\right) \pi=\left(1-s_{k}\right)(1-z) Y \\
=\left(1-s_{k}\right)(1-z) a K
\end{gathered}
$$

where $z$ is the share of wages in national income, $s_{k}$ is the capitalists' average propensity to save, and $\pi$ is the real profit. Following Lancaster (1973), Hoel (1978), Pohjola (1983), Mehrling (1986), Ishigaki (1994) and Asada (1997), we assume that $z$ is controlled by workers and $s_{k}$ is controlled by capitalists.

Also in this section, we adopt the following specification of the functional forms.

$$
\begin{gathered}
U_{1}\left(C_{W} / N\right)=\log \left(C_{W} / N\right) \\
V_{1}(P)=\alpha P ; \quad \alpha>0 \\
U_{2}\left(C_{K} / K\right)=\log \left(C_{K} / K\right) \\
\varphi(g)=\beta(1+g) ; \quad \beta>0
\end{gathered}
$$

In this case, $W_{1}$ and $W_{2}$ become as follows:

$$
\begin{aligned}
& W_{1}\left(z(t), s_{k}(t)\right) \equiv \int_{0}^{\infty} f_{1}\left(z(t), s_{k}(t) ; \alpha \beta\right) K(t) \exp (-\rho t) \mathrm{d} t \\
& W_{2}\left(z(t), s_{k}(t)\right) \equiv \int_{0}^{\infty} f_{2}\left(z(t), s_{k}(t)\right) K(t) \exp (-\rho t) \mathrm{d} t
\end{aligned}
$$

where,

$$
\begin{gathered}
f_{1}\left(z(t), s_{k}(t) ; \alpha \beta\right) \equiv[\log z(t)+\log a-\log b \\
\left.-(\alpha \beta / b)\left\{1+s_{k}(t)(1-z(t)) a\right\}\right] b \\
f_{2}\left(z(t), s_{k}(t)\right) \equiv \log \left\{1-s_{k}(t)\right\}+\log \{1-z(t)\} \\
+\log a .
\end{gathered}
$$

\footnotetext{
${ }^{* *}$ The formal structure of our model is similar to the so called "AK model" in the endogenous growth theory in the sense that the rate of economic growth is proportional to the social rate of saving, and it is endogenously determined. See, for example, Barro and Sala-i-Martin (1995).

${ }^{\dagger+}$ Analytical framework of the model presented in this section is essentially based on Ishigaki (1994) and Asada (1997). In their approaches, however, the environmental factors are not introduced contrary to the present version.

"I In this formulation, we follow the hypothesis of the "spirit of capitalism" which was introduced by Robson (1992) and Bakshi and Chen (1996). In their formulation, capitalists' utility depends not only on their consumption but also on their social standing which is symbolized by their wealth. In this model, their wealth is represented by the real capital stock $(K)$ and it is assumed that capitalists' utility is linear homogeneous with respect to $C_{K}$ and $K$.
} 
Both classes are subject to the common dynamic constraint which is given by

$$
\begin{gathered}
\dot{K}(t)=s_{k}(t)\{1-z(t)\} a K(t), \\
K(0) \equiv K_{0}=\text { given. }>0 .
\end{gathered}
$$

Now, let us consider the simplest solution concept of the (noncooperative) differential game, i.e. the "open loop Nash solution". It is defined as the path $\left(\hat{z}(t), \hat{s}_{k}(t)\right) \quad(t \in[0, \infty))$ which satisfies the following conditions for all piecewise continuous paths with respect to time, $\left(z(t), s_{k}(t)\right) \quad(t \in[0, \infty)) .^{\S \S}$

$$
\begin{aligned}
\text { (i) } & W_{1}\left(\hat{z}(t), \hat{s}_{k}(t)\right) \geqq W_{1}\left(z(t), \hat{s}_{k}(t)\right) \\
\text { (ii) } & W_{2}\left(\hat{z}(t), \hat{s}_{k}(t)\right) \geqq W_{2}\left(\hat{z}(t), s_{k}(t)\right)
\end{aligned}
$$

We can solve such a problem by applying Pontryagin's maximum principle. Workers' current value Hamiltonian $\left(H_{1}(t)\right)$ is defined as

$$
\begin{aligned}
H_{1}(t) \equiv & f_{1}\left(z(t), s_{k}(t) ; \alpha \beta\right) K(t)+\lambda_{1}(t) s_{k}(t)\{1 \\
& -z(t)\} a K(t)
\end{aligned}
$$

where $\lambda_{1}(t)$ is workers' costate variable. Then, we can formulate a set of necessary conditions of optimality for workers as follows:

i)

$$
\operatorname{Max}_{z(t) \in[0,1]} H_{1}(t) \text { for all } t \geqq 0
$$

ii)

$$
\dot{\lambda}_{1}(t)=-\partial H_{1}(t) / \partial K(t)+\rho \lambda_{1}(t) \text { for all } t \geqq 0
$$

iii)

$$
\lim _{t \rightarrow \infty} \lambda_{1}(t) \exp (-\rho t)=0 .
$$

On the other hand, capitalists' current value Hamiltonian $\left(H_{2}(t)\right)$ is

$$
\begin{aligned}
H_{2}(t) \equiv & f_{2}\left(z(t), s_{k}(t)\right) K(t)+\lambda_{2}(t) s_{k}(t)\{1 \\
& -z(t)\} a K(t)
\end{aligned}
$$

where $\lambda_{2}(t)$ is capitalists' costate variable, and a set of necessary conditions of optimality for capitalists is expressed as follows:

$$
\text { (i) } \underset{s_{k}(t) \in[0,1]}{\operatorname{Max}} H_{2}(t) \text { for all } t \geqq 0
$$

(ii) $\quad \dot{\lambda}_{2}(t)=-\partial H_{2}(t) / \partial K(t)+\rho \lambda_{2}(t)$ for all $t \geqq 0$

$$
\text { (iii) } \lim _{t \rightarrow \infty} \lambda_{2}(t) \exp (-\rho t)=0 \text {. }
$$

First, let us consider the solution of workers' problem. We have

$$
\begin{gathered}
\partial H_{1}(t) / \partial z(t)=\left[b / z(t)+\alpha \beta a-\lambda_{1}(t) s_{k}(t) a\right] K(t), \\
\partial^{2} H_{1}(t) / \partial z(t)^{2}=\left\{-b / z(t)^{2}\right\} K(t)<0 .
\end{gathered}
$$

If we assume the internal solution, Eq. (50)(i) becomes $\partial H_{1}(t) / \partial z(t)=0$, so that we obtain

$$
\begin{aligned}
\lambda_{1}(t) & =(b / a) / z(t) s_{k}(t)+\alpha \beta / s_{k}(t) \equiv \lambda_{1}\left(z(t), s_{k}(t)\right) \\
& >0 \text { for } s_{k}(t) \in(0,1] .
\end{aligned}
$$

Substituting Eq. (55) into Eq. (50)(ii), we have

$$
\begin{gathered}
-\left\{(b / a) / z(t)^{2} s_{k}(t)\right\} \dot{z}(t)-\left\{(b / a) / z(t) s_{k}(t)^{2}\right. \\
\left.+\alpha \beta / s_{k}(t)^{2}\right\} \dot{s}_{k}(t)=-f_{1}\left(z(t), s_{k}(t) ; \alpha \beta\right) \\
+\left[\rho-s_{k}(t)\{1-z(t)\} a\right] \lambda_{1}\left(z(t), s_{k}(t)\right) \\
\equiv F_{1}\left(z(t), s_{k}(t) ; \alpha \beta\right) .
\end{gathered}
$$

If $s_{k}(t)$ is fixed throughout time, the stationary solution of Eq. (56) is given by

$$
F_{1}\left(z(t), s_{k}(t) ; \alpha \beta\right)=0 .
$$

We obtain the following relationships.

$$
\begin{aligned}
A \equiv & \partial F_{1} / \partial z(t) \\
= & \alpha \beta a\{1-z(t)\}-\left[\rho-s_{k}(t)\{1\right. \\
& -z(t)\} a]\left\{b / z(t)^{2}\right\}
\end{aligned}
$$

$B \equiv \partial F_{1} / \partial s_{k}(t)$

$$
\begin{gathered}
=\alpha \beta a\{1-z(t)\}-\left\{\rho / s_{k}(t)^{2}\right\}\{(b / a) / z(t)+\alpha \beta\} \\
E \equiv \partial F_{1} / \partial(\alpha \beta)=1+s_{k}(t)\{1-z(t)\} a>0 \\
\lim _{z(t) \rightarrow 0} F_{1}\left(z(t), s_{k}(t) ; \alpha \beta\right)=-\left[\lim _{z(t) \rightarrow 0} \log z(t)+\log a\right. \\
\left.-\log b-(\alpha \beta / b)\left\{1+s_{k}(t) a\right\}\right] b \\
+\left\{\rho / s_{k}(t)-a\right\}\left\{\lim _{z(t) \rightarrow 0}(b / a) / z(t)+\alpha \beta\right\} \\
F_{1}\left(1, s_{k}(t) ; \alpha \beta\right)=\left\{\log (b / a)+\rho / s_{k}(t) a\right\} b+\alpha \beta\{1 \\
\left.+\rho / s_{k}(t)\right\}
\end{gathered}
$$

Now, let us assume as follows:

Assumption 2 (i) $\rho>a$ (ii) $A<0$ (iii) $B<0$ (iv) $F_{1}(1,1, \alpha \beta)=\{\log (b / a)+\rho / a\} b+\alpha \beta(1+\rho)<0$

\footnotetext{
${ }^{\S}$ See, for example, Bagchi (1984) and Basar and Olsder (1995) as for the related topics. See also Asada (1997) and Asada (1999).
} 


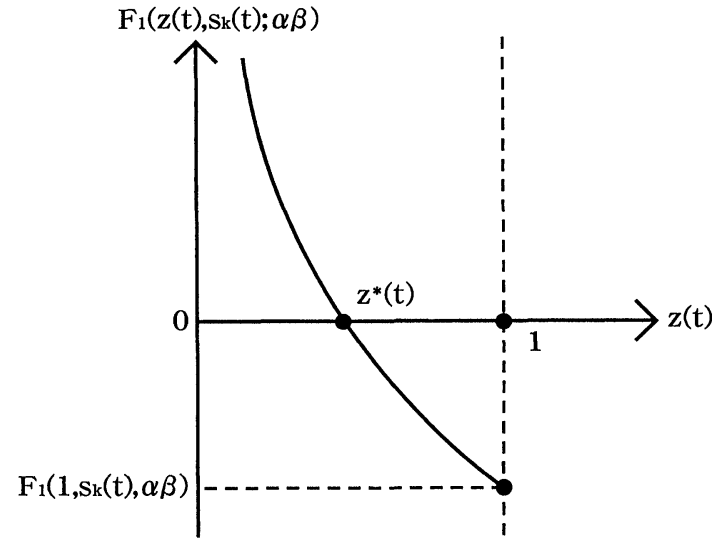

FIGURE 2 Solution of Eq. (57).

Assumption 2(i) and 2(iv) are in fact equivalent to Assumption 1(i) and 1(ii) in the previous section. Assumption 2(ii) and 2(iii) will be satisfied if $\alpha \beta$ is relatively small.

It follows from Assumption 2(i) that

$$
\lim _{z(t) \rightarrow 0} F_{1}\left(z(t), s_{k}(t) ; \alpha \beta\right)=+\infty \text { for } s_{k}(t) \in(0,1] .
$$

On the other hand, Assumption 2(iv) implies that there exists the level of the capitalists' saving propensity $\tilde{s_{k}} \in(0,1)$ such that

$$
F_{1}\left(1, s_{k}(t) ; \alpha \beta\right)<0 \text { for all } s_{k}(t) \in\left(\tilde{s_{k}}, 1\right] .
$$

It is clear that under Assumption 2, there exists the unique solution $z(t) \in(0,1)$ of Eq. (57) when $s_{k}(t)$ is fixed at the level $s_{k}(t) \in\left(\tilde{s}_{k}, 1\right]$ (see Fig. 2). In this case, we can express the solution of Eq. (57) as follows:

$$
\begin{gathered}
z(t)=\Phi_{1}\left(s_{k}(t) ; \alpha \beta\right) ; \quad \partial \Phi_{1} / \partial s_{k}=-B / A<0, \\
\partial \Phi_{1} / \partial(\alpha \beta)=-E / A>0, \\
\partial^{2} \Phi_{1} / \partial s_{k}=\left(-B^{\prime} A+B A^{\prime}\right) / A^{2},
\end{gathered}
$$

where $A^{\prime} \equiv \mathrm{d} A / \mathrm{d} s_{k}(t)$ and $B^{\prime} \equiv \mathrm{d} B / \mathrm{d} s_{k}(t)$.

We can prove that the workers' optimal policy is in fact given by Eq. (65) when $s_{k}(t) \in\left(\tilde{s_{k}}, 1\right]$ is fixed. " "We can consider the function $\Phi_{1}\left(s_{k}(t) ; \alpha \beta\right)$ as workers' response function. Figure 3 is an example of such a function. ${ }^{\# \#}$ It is clear that the function $\Phi_{1}$ in Fig. 3 shifts upward when $\alpha \beta$ increases.

Next, let us solve the capitalists' problem. From Eqs. (46) and (51) we have

and

$$
\begin{gathered}
\partial H_{2}(t) / \partial s_{k}(t)=\left[-1 /\left\{1-s_{k}(t)\right\}\right. \\
\left.+\lambda_{2}(t)\{1-z(t)\} a\right] K(t)
\end{gathered}
$$

$$
\partial^{2} H_{2}(t) / \partial s_{k}(t)^{2}=-K(t) /\left\{1-s_{k}(t)\right\}^{2}<0 .
$$

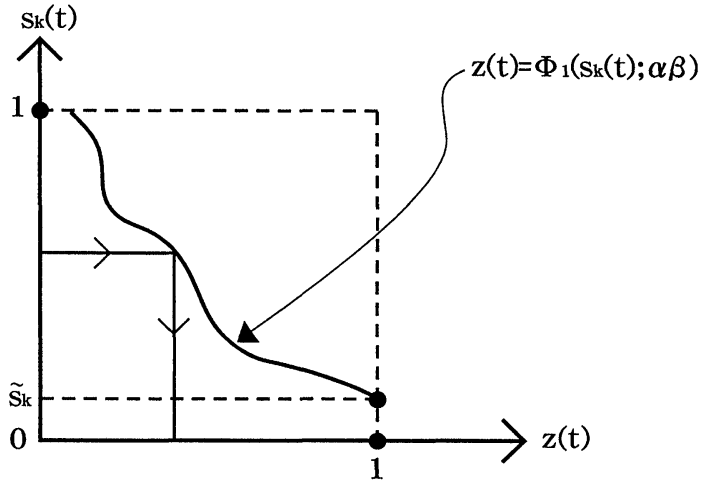

FIGURE 3 Workers' response function.

Therefore, the first order condition of Eq. (52)(i) becomes

$$
\begin{aligned}
\lambda_{2}(t) & =1 /\left[\left\{1-s_{k}(t)\right\}\{1-z(t)\} a\right] \equiv \lambda_{2}\left(z(t), s_{k}(t)\right) \\
& >0 .
\end{aligned}
$$

Substituting Eq. (68) into Eq. (52)(ii), we have

$$
\begin{aligned}
\dot{z}(t) /[ & \left.\left\{1-s_{k}(t)\right\}\{1-z(t)\}^{2} a\right] \\
+ & \dot{s}_{k}(t) /\left[\left\{1-s_{k}(t)\right\}^{2}\{1-z(t)\} a\right] \\
& =-f_{2}\left(z(t), s_{k}(t)\right) \\
+ & {\left[\rho-s_{k}(t)\{1-z(t)\} a\right] \lambda_{2}\left(z(t), s_{k}(t)\right) } \\
& \equiv F_{2}\left(z(t), s_{k}(t)\right) .
\end{aligned}
$$

If $z(t)$ is fixed throughout time, the stationary solution of Eq. (69) is given by

Assumption 3

$$
F_{2}\left(z(t), s_{k}(t)\right)=0 .
$$

$$
F_{2}(0,0)=-\log a+\rho / a<1
$$

Assumption 3 together with Assumption 2(i) implies that $a<\rho<a \log a$, which is possible only if $\log a>1$, in other words, $a>e$. We can easily show that under Assumption 3, there exists the level of wage share $\tilde{z} \in(0,1)$ such that the solution $s_{k}(t) \in(0,1)$ of Eq. (70) is uniquely determined by the following equation for $z(t) \in[0, \tilde{z})$.

$$
s_{k}(t)=\Phi_{2}(z(t)) ; \quad \Phi_{2}^{\prime}(z(t))<0
$$

Equation (71) is capitalists' response function, which gives the capitalists' optimal policy when $z(t) \in[0, z)$ is fixed. Figure 4 is an example of such a function. The combination $\left(z^{*}, s_{k}^{*}\right)$ which corresponds to the intersection of workers' response function $\left(\Phi_{1}\right)$ and capitalists' response function $\left(\Phi_{2}\right)$ gives the open loop Nash solution in this system, and the equilibrium rate of economic growth $\left(g^{*}\right)$ is endogenously determined by

\footnotetext{
११ The method of the proof of this proposition is almost the same as that of the proof of Proposition 1.

${ }^{\# \#}$ In general, the sign of $\partial^{2} \Phi_{1} / \partial s_{k}(t)^{2}$ is indeterminate.
} 


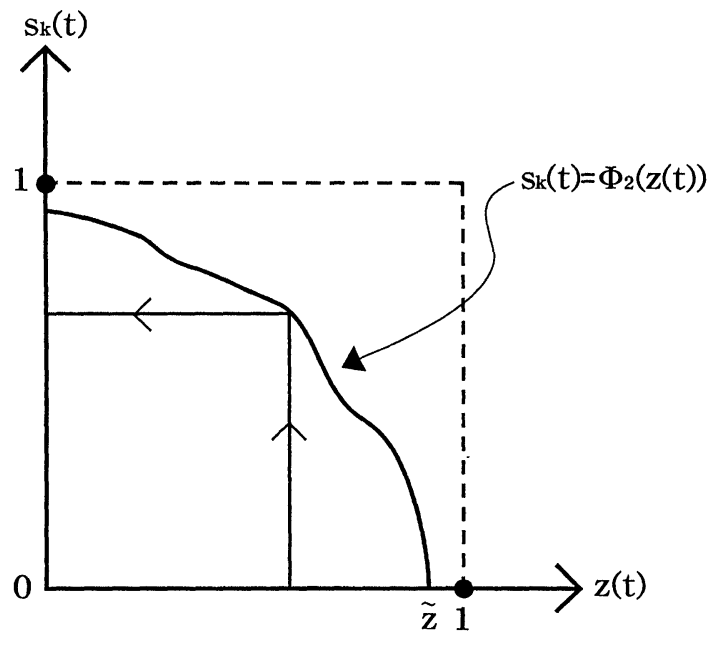

FIGURE 4 Capitalists' response function.

$$
g^{*}=\dot{K} / K=s_{k}^{*}\left(1-z^{*}\right) a>0
$$

correspondingly.

Proposition 3 The open loop Nash solution of the noncooperative differential game which is formulated in this section is also the feedback Nash solution. In other words, it is subgame perfect (time consistent). ${ }^{* * *}$

Proof Two response functions $\left(\Phi_{1}\right.$ and $\left.\Phi_{2}\right)$ are independent of the state variable $K(t)$, i.e. this system is "state separable". Therefore, both players (workers and capitalists) have no incentive to change their strategies $\left(z^{*}, s_{k}{ }^{*}\right)$ even if they are allowed to replay the game which starts at the arbitrary period $\tau>0$.

Henceforth, we shall call the open loop Nash solution of this system as the "equilibrium solution" for simplicity. In general, there exist the multiple equilibrium solutions.

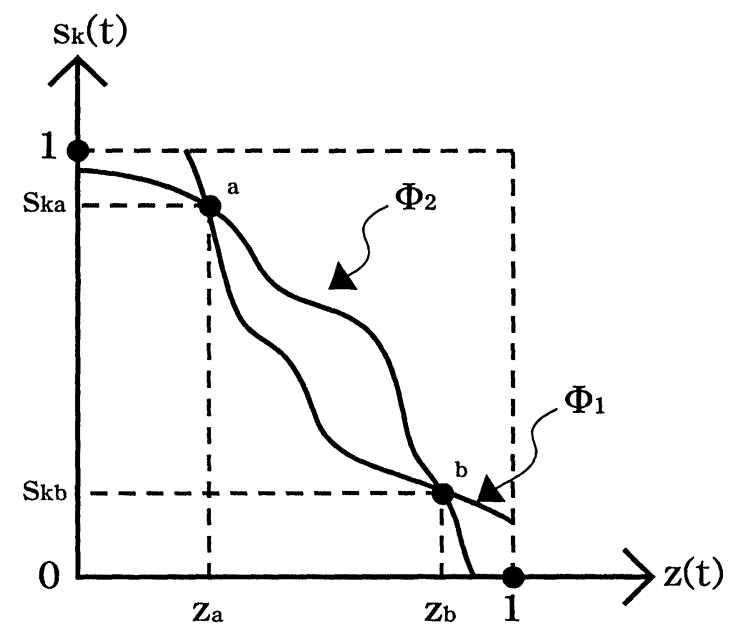

FIGURE 5 Equilibrium solutions.

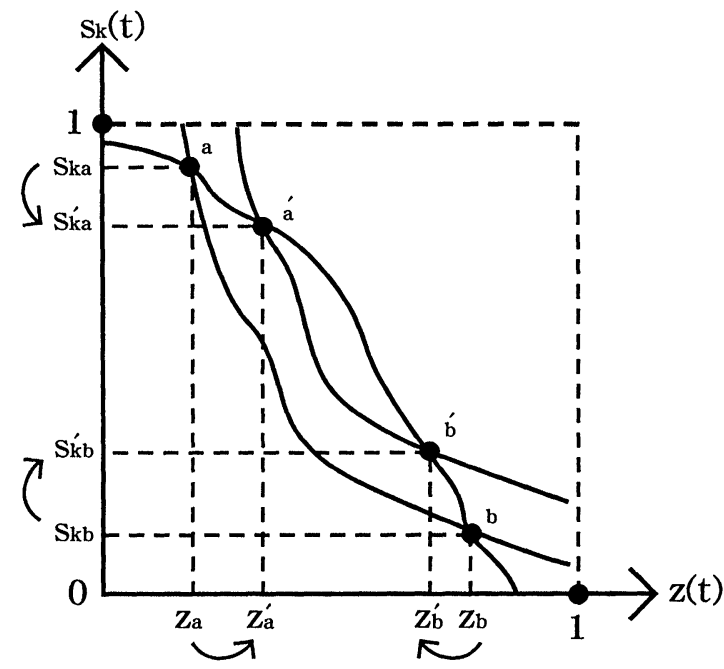

FIGURE 6 Comparative dynamic analysis.

Figure 5 shows the case in which two equilibrium solutions exist. Point $a$ in this figure corresponds to the solution with high rate of growth and high level of pollution. On the other hand, point $b$ is the solution with low rate of growth and low level of pollution.

In general, there is no reason to exclude a priori one of such equilibrium solutions. In such a case, we can obtain some counterintuitive results. For example, suppose that $\alpha \beta$ increases. This means the increase of workers' disutility of pollution $(\alpha)$ or the increase of the adverse pollution effect of capital accumulation $(\beta)$. Intuitively, it may be expected that the equilibrium growth rate must decrease correspondingly. However, this is not necessarily the case. Figure 6 shows that the intuition is not supported for the solution $b$ (solution with low growth rate and low pollution) although the intuition is supported for the solution a (solution with high growth rate and high pollution). ${ }^{\dagger \dagger}$ This result seems to suggest that the realistic "decentralized" solution with the conflict of interests between different agents is much more complicated than the "collective" solution which is simply formulated in terms of the optimization problem of the single agent.

\section{CONCLUDING REMARKS}

In this paper, we have studied two dynamic models of capital accumulation with environmental factors, and investigated the economic implications of the trade off between growth and environment. In particular, we have shown that the differential game approach can contribute to reveal some aspects which have been rather neglected so far in the environmental economics. In this paper, however, we have not considered the economic activity that eliminates the environmental pollution although we

\footnotetext{
${ }^{* * *}$ As for the concept of the feedback Nash solution, see Bagchi (1984), Basar and Olsder (1995) and Ishigaki (1994).

${ }^{+\dagger+}$ In Appendix B, we present a tatonnement process in which the point $a$ is stable and the point $b$ is unstable. However, we must not interpret this process as the actual development through time. Therefore, it is not the reliable foundation to exclude the "counterintuitive" equilibrium solution.
} 
have introduced the adverse effect of economic growth on the environment. Economic analysis of the activity which eliminates the environmental pollution in the dynamic context is the theme which is left to the study in future.

\section{Acknowledgements}

This research was financially supported by CRUGE (Chuo Research Unit for Global Environment) which is founded in Chuo University, Tokyo, Japan and the Japan Society for the Promotion of Science.

\section{References}

Asada, T. (1997) Macrodynamics of Growth and Cycles (Nihon Keizai Hyoron-sha, Tokyo), in Japanese.

Asada, T. (1999) "A differential game approach to transportation competition", Journal of Economics of Chuo University 39(3-4), 15-28, Tokyo, Japan.

Bagchi, A. (1984) Stackelberg Differential Games in Economic Models (Springer, Berlin).

Bakshi, G.S. and Chen, Z. (1996) "The spirit of capitalism and stockmarket prices", American Economic Review 86-1, 133-157.

Barro, R.J. and Sala-i-Martin, X. (1995) Economic Growth (McGrawHill, New York).

Basar, T. and Olsder, G.J. (1995) Dynamic Noncooperative Game Theory (Academic Press, New York).

Chiang, A.C. (1992) Elements of Dynamic Optimization (McGraw-Hill, New York).

Clark, C.W. (1976) Mathematical Bioeconomics (Wiley, New York).

Dockner, E. and van Long, N. (1993) "International pollution control: cooperative versus noncooperative strategies", Journal of Environmental Economics and Management 24, 13-29.

Dockner, E., Feichtinger, G. and Mehlmann, A. (1989) "Noncooperative solutions for a differential game model of fishery", Journal of Economic Dynamics and Control 13, 1-20.

Forster, B.A. (1980) "Optimal energy use in a polluted environment", Journal of Environmental Economics and Management, 321-333.

Hartl, R.F. and Kort, P.M. (1996) "Capital accumulation of a firm facing an emissions tax", Journal of Economics/Zeitschrift für Nationalökonomie 63-1, 1-23.

Hettich, F. (2000) Economic Growth and Environmental Policy: A Theoretical Approach (Edward Elgar, Cheltenham, UK).

Hoel, M. (1978) "Distribution and growth as a differential game between workers and capitalists", International Economic Review 19, $335-350$.

Inoue, T. (1998) "A survey of analysis of the transboundary pollution problem: symmetric and asymmetric dynamic models", Studies in Regional Science 28-2, 19-39.

Ishigaki, T. (1994) "Workers' and capitalists' cooperativeness and capital accumulation: an examination of differential game models", The Hitotsubashi Review 112-6, 135-154, (Tokyo, Japan) (in Japanese).

Keeler, E.M., Spence, M. and Zeckhauser, R. (1971) "The optimal control of pollution", Journal of Economic Theory 4, 19-34.

Lancaster, K. (1973) "The dynamic inefficiency of capitalism", Journal of Political Economy 81, 1092-1109.

Mäler, K.G. (1974) Environmental Economics, A Theoretical Inquiry (John Hopkins University Press, Baltimore).

Mäler, K.G. and de Zeeuw, A. (1998) "The acid rain differential game", Environmental and Resource Economics 12, 167-184.

Mehrling, P.G. (1986) "A classical model of the class struggle: a gametheoretic approach", Journal of Political Economy 94, 1280-1303.

Pohjola, M. (1983) "Nash and stackelberg solution in a differential game model of capitalism", Journal of Economic Dynamics and Control 6, $173-186$.

Robson, A.J. (1992) "Status, the distribution of wealth, and social attitudes to risk", Econometrica 60-4, 837-857.
Stimming, M. (1999) “Capital accumulation subject to pollution control: open-loop versus feedback investment strategies", Annals of Operations Research 88, 309-336.

Uzawa, H. (1995) Economic Analysis of Global Warming (Iwanami Shoten (in Japanese), Tokyo).

Uzawa, H., (1999). "Global Warming and Economic Theory." Chuo Research Unit for Global Environment (CRUGE), Chuo University, Tokyo, Japan (unpublished).

\section{APPENDIX A}

Let us write the path of $[f(s(t) ; \alpha \beta), K(t), s(t)]$ which satisfies Eq. (25) in the text as $\left[f^{*}, K(t)^{*}, s^{*}\right]$, and write the arbitrary path of $[f(s(t) ; \alpha \beta), K(t), s(t)]$ which satisfies the piecewise continuity with respect to time and the condition $s(t) \in[0,1]$ as $[\tilde{f}(t), \tilde{K}(t), \tilde{s}(t)]$. (We assume that all paths depart from the same initial condition $K(0)=K_{0}$.). Then, we obtain the following equality.

$$
\begin{aligned}
M \equiv & \int_{0}^{\infty}\left[f^{*} K(t)^{*}-\tilde{f}(t) \tilde{K}(t)\right] \exp (-\rho t) \mathrm{d} t \\
= & \int_{0}^{\infty}\left[f^{*} K(t)^{*}-\tilde{f}(t) \tilde{K}(t)+\lambda^{*}\left\{s^{*} a K(t)^{*}\right.\right. \\
& \left.-\dot{K}(t)^{*}\right\}-\lambda^{*}\{\tilde{s}(t) a \tilde{K}(t) \\
& -\dot{\tilde{K}}(t)\}] \exp (-\rho t) \mathrm{d} t \\
= & \int_{0}^{\infty}\left[f^{*} K(t)^{*}-\tilde{f}(t) \tilde{K}(t)+\lambda^{*} s^{*} a K(t)^{*}\right. \\
& \left.-\lambda^{*} \tilde{s}(t) a \tilde{K}(t)\right] \exp (-\rho t) \mathrm{d} t \\
& +\left[\lambda^{*} \exp (-\rho t)\left\{\tilde{K}(t)-K(t)^{*}\right\}\right]_{t=0}^{t=\infty} \\
& -\int_{0}^{\infty}\left\{\lambda^{*} \exp (-\rho t)\right\}\left\{\tilde{K}(t)-K(t)^{*}\right\} \mathrm{d} t ;
\end{aligned}
$$

where $\lambda^{*} \equiv \lambda\left(s^{*}\right)=(b / a) /\left(1-s^{*}\right)+\alpha \beta>0$. We have

$$
\begin{aligned}
& {\left[\lambda^{*} \exp (-\rho t)\left\{\tilde{K}(t)-K(t)^{*}\right\}\right]_{t=0}^{t=\infty}} \\
& \quad=\lim _{t \rightarrow \infty}\left[\lambda^{*} \exp (-\rho t)\left\{\tilde{K}(t)-K(t)^{*}\right\}\right] \\
& \quad-\lambda^{*}\left\{K_{0}-K_{0}\right\} \\
& \quad=\lambda^{*} K_{0} \lim _{t \rightarrow \infty} \exp \int_{0}^{t}(\tilde{s}(\tau) a-\rho) d \tau \\
& \quad-\lim _{t \rightarrow \infty} \exp \left\{\left(s^{*} a-\rho\right) t\right\}=0
\end{aligned}
$$

because $0 \leqq \tilde{s}(t) \leqq 1,0<s^{*}<1$ and $a<\rho$ from Assumption 1. We also have from Eq. (16)(ii) in the text that

$$
\begin{aligned}
\left\{\lambda^{*} \exp (-\rho t)\right\} & =-\{\partial H(t) / \partial K(t)\} \exp (-\rho t) \\
& =-\left(f^{*}+s^{*} a\right) \exp (-\rho t) .
\end{aligned}
$$


Substituting Eq. (A2) and Eq. (A3) into Eq. (A1), we obtain

$$
\begin{aligned}
M= & \int_{0}^{\infty}\left\{\left(f^{*}+\lambda^{*} s^{*} a\right)-(\tilde{f}(t)\right. \\
& \left.\left.+\lambda^{*} \tilde{s}(t) a\right)\right\} \tilde{K}(t) \exp (-\rho t) \mathrm{d} t .
\end{aligned}
$$

Let us define the function $G(s(t))$ as

$$
G(s(t)) \equiv f(s(t))+\lambda^{*} s(t) a .
$$

Then, the function $G(s(t))$ becomes a strict concave function since $G^{\prime \prime}(s(t))=f^{\prime \prime}(s(t))=-b /\{1-s(t)\}^{2}<0$. Therefore, we have the following relationship because of the nature of the concave function.

$$
G\left(s^{*}\right)-G(\tilde{s}(t)) \geqq G^{\prime}\left(s^{*}\right)\left\{s^{*}-\tilde{s}(t)\right\}
$$

where the equality in Eq. (A6) is satisfied if and only if $s^{*}=\tilde{s}(t)$. On the other hand, it follows from Eq. (19) in the text that

$$
G^{\prime}\left(s^{*}\right)=f^{\prime}\left(s^{*}\right)+\lambda^{*} s^{*} a=0 .
$$

From Eqs. (A4), (A6) and (A7) we obtain

$$
M \equiv \int_{0}^{\infty}\left\{f^{*} K^{*}-\tilde{f}(t) \tilde{K}(t)\right\} \exp (-\rho t) \mathrm{d} t \geqq 0
$$

where the strict inequality in Eq. (A8) is satisfied if $\tilde{s}(t) \neq$ $s^{*}$ for some $t \geqq 0$ because of the piecewise continuity of $\tilde{s}(t)$ with respect to $t$. This completes the proof of Proposition 1.

\section{APPENDIX B}

Let us reformulate the response functions of two classes $z(t)=\Phi_{1}\left(s_{k}(t) ; \alpha \beta\right)$ and $s_{k}(t)=\Phi_{2}(z(t))$ as the following

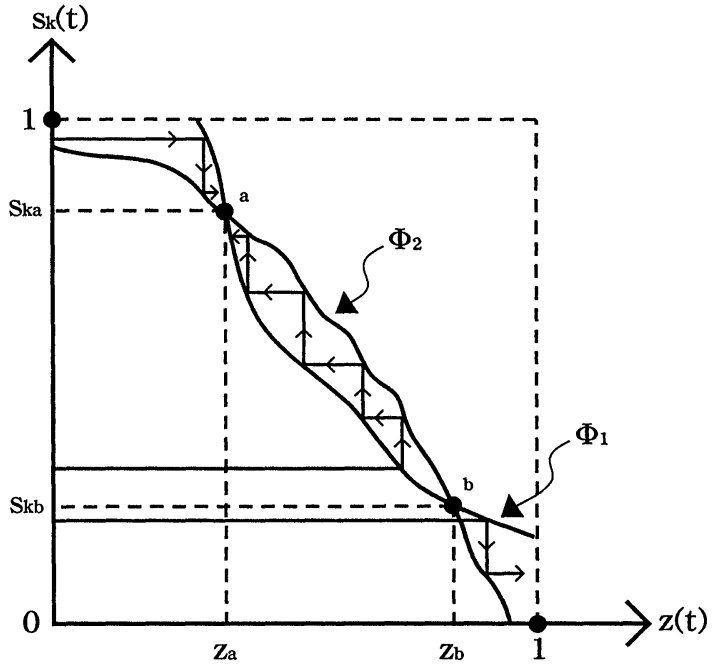

FIGURE 7 Stability of the hypothetical adjustment.

discrete dynamical system with one period time lag.

$$
\begin{gathered}
\ldots, z(t)=\Phi_{1}\left(s_{k}(t-1) ; \alpha \beta\right), \\
s_{k}(t+1)=\Phi_{2}(z(t)), \\
z(t+2)=\Phi_{1}\left(s_{k}(t+1) ; \alpha \beta\right), \\
s_{k}(t+3)=\Phi_{2}(z(t+2)), \ldots
\end{gathered}
$$

We suppose that these adjustments do not occur simultaneously, but they occur sequentially. We must interpret this adjustment process as the tatonnement algorithm that tries to solve the equilibrium solution by iteration rather than the picture of the actual development through time. As Fig. 7 shows, under this (hypothetical) adjustment process, the point a (high growth, high pollution equilibrium) is stable, while the point $b$ (low growth, low pollution equilibrium) is unstable. 


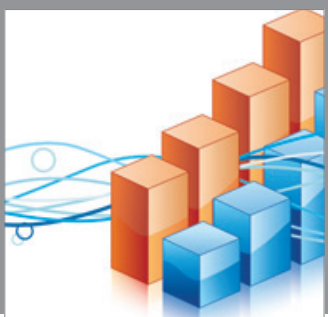

Advances in

Operations Research

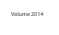

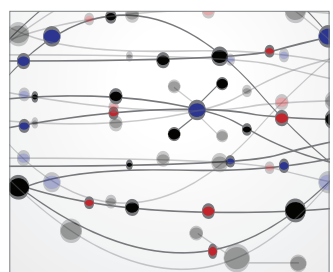

\section{The Scientific} World Journal
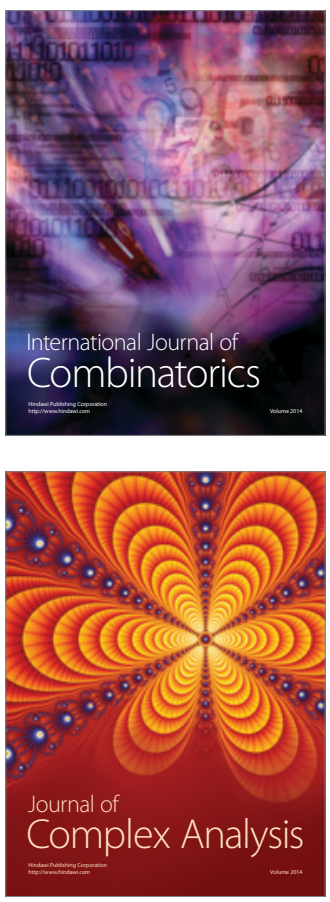

International Journal of

Mathematics and

Mathematical

Sciences
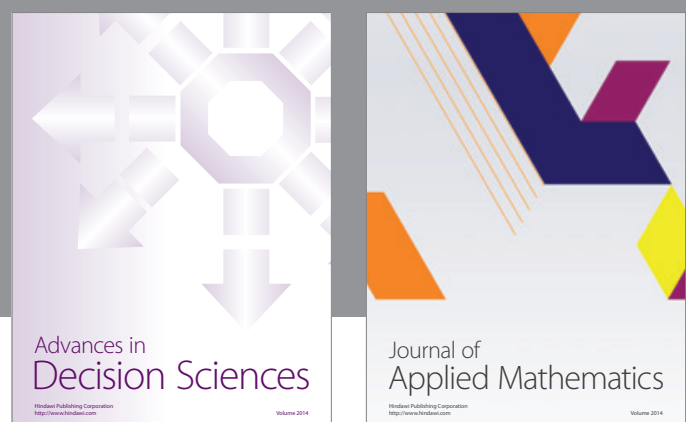

Journal of

Applied Mathematics
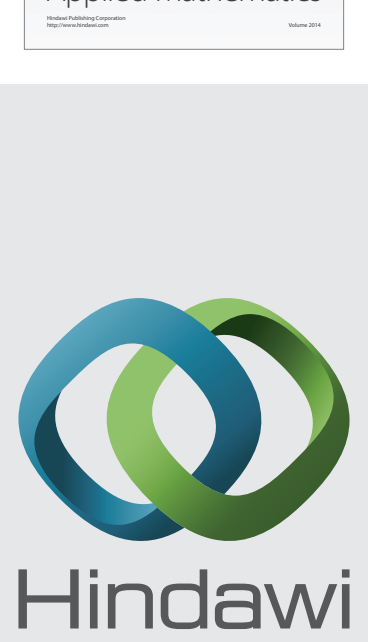

Submit your manuscripts at http://www.hindawi.com
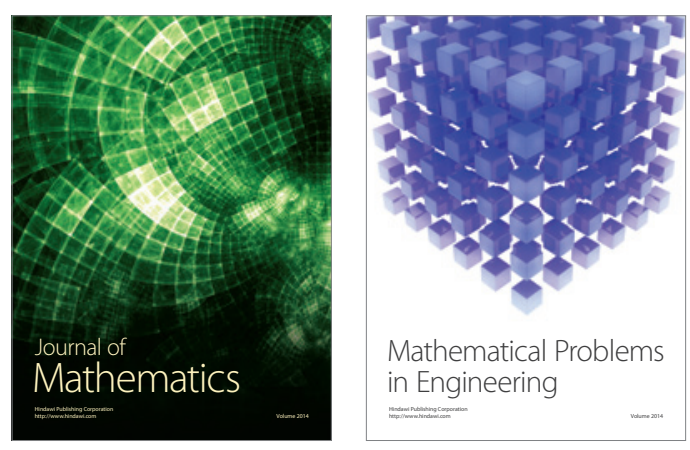

Mathematical Problems in Engineering
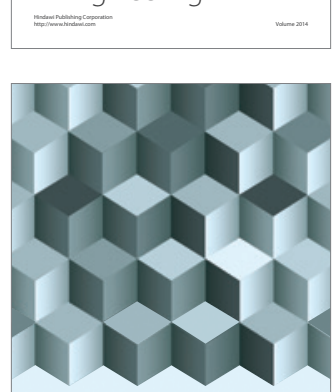

Journal of

Function Spaces
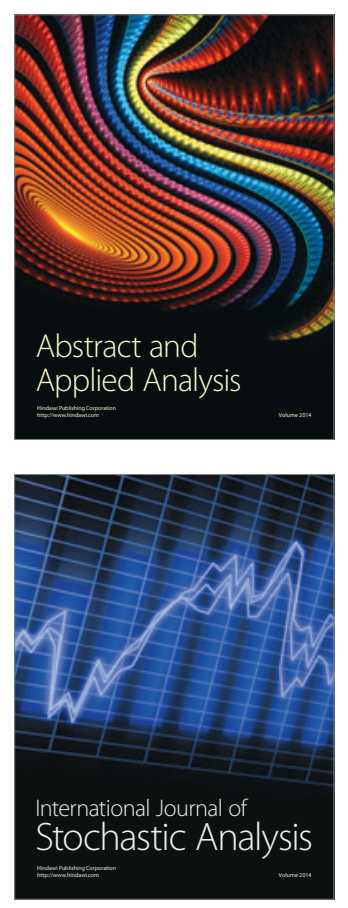

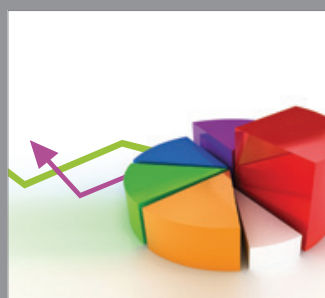

ournal of

Probability and Statistics

Promensencen
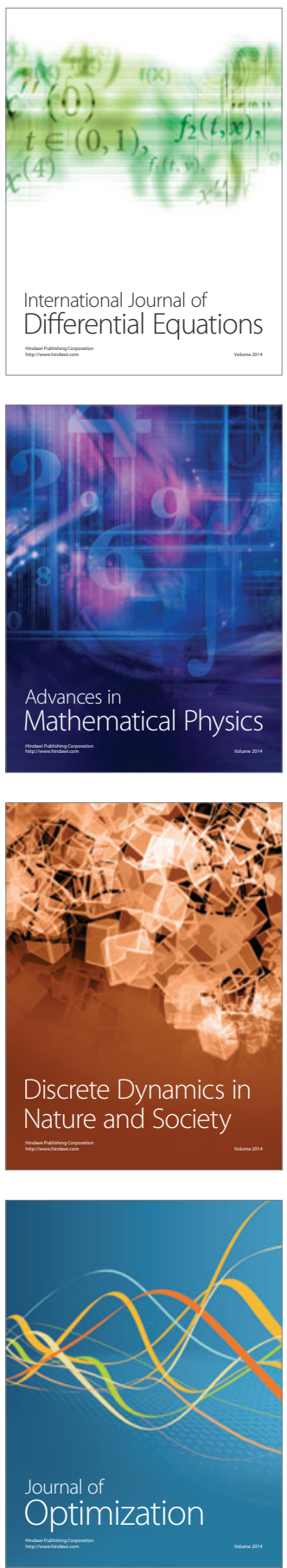